\title{
Evolution of Diversity Gain with and without Coding Gain in MIMO for Emerging Wireless Networks
}

\author{
Naveed Ali Kaimkhani, Zhe Chen, and Fuliang Yin
}

\begin{abstract}
The wireless networks are growing rapidly that cause constraint in data rate and power consumption. The multiple-input multiple-output (MIMO) has incredible performance to overcome the limitations by spatial multiplexing gain. Spatial multiplexing gain is achieved with the help of space time encoding technique in which a high rate signal splitting into multiple lower rate streams and transmitting them through the different antennas. This paper provides a comprehensive comparative study of diversity gain with and without coding gain for $4 \times 2$ and $4 \times 4$ MIMO systems. The coding gain is explored through turbo coding (TC) with $1 / 2$ and $3 / 4$ coding rates, and diversity gain is adopted by orthogonal space time coding (OSTBC). Turbo coding is useful to reduce the forward error correction in multiplexed data transmissions. The compounding of OSTBC and turbo coding is valuables to increase data rate and reduce energy consumption by reducing bit error rate in emerging wireless network transmissions. Simulation results convinced the validity of proposed scheme.
\end{abstract}

Index Terms-Emerging wireless networks, OSTBC, turbo coding, diversity and coding gain, MIMO.

\section{INTRODUCTION}

The multiple-input multiple-output (MIMO) is commonly used in wireless communication due to capability of user effective multi-path propagation, to improve overall capacity and spectrum efficiency through antenna diversity scheme. Therefore, space time block coding (STBC) is well acknowledged as Almaouti's scheme has been very effective to provide diversity gain in wireless attenuated channels [1]. The turbo code (TC) is very effective to manage fading, noise and interference to acquire coveted gain with the help of interleaving [2]. The forward error correction method enlighten by TC is used to improve the performance with STBC in MIMO networks. And also the combination of STBC and turbo code is helpful to obtain diversity gain and coding gain respectively in MIMO channel.

The researchers have been enlightened the various combinations of channel coding with antenna diversity to improve the performance of new emerging wireless networks. The powerful channel code method well known as turbo code with the combination of STBC to obtain coding and diversity gains is proposed in [3]-[5] proposed. Additionally, in [6] the

Manuscript received May 5, 2015; revised November 15, 2015. This work was acknowledged by National Natural Science Foundation of China (No.61172107, No.61172110), Dalian Municipal Science and Technology Fund Scheme of China (No. 2008J23JH025), Specialized Research Fund for the Doctoral Program of Higher Education of China (No. 200801410015) and the fundamental research funds for the central university of China (DUT13LAB06).

All authors are with the School of Information and Communication Engineering, Dalian University of Technology, Dalian, 116023 P.R. China (e-mail: naveedali64@yahoo.fr, zhechen@dlut.edu.cn, flyin@dlut.edu.cn). combination of turbo and STBC codes to improve receiver performance against channel fading channel was discussed with the help of iterative decoding method.

The turbo and STBC codes in CDMA performance are also appraised through minimizing complexity of receiver for multi-users [7]. In [8], a multistage receiver was designed with the help of MMSE and interference cancellation method. Furthermore, in [9] the low density soft iterative multi-user through multi user detection (MUD) and MMSE detector was discussed.

The performance of orthogonal space time block coding (OSTBC) with and without coding gain (turbo code) in MIMO channel for BPSK, QPSK and 8-PPSK modulation scheme is evaluated. Furthermore, the study is also sustained for turbo code $1 / 2$ and $3 / 4$ rates to assess the coding gain performance in $4 \times 2$ and $4 \times 4$ MIMO. Motivation of proposed scheme is to understand the performance of diversity gain by OSTBC with coding gain through turbo code to improve the emerging wireless networks. As OSTBC is useful to handle the power consumption and decrease the signal to noise ratio through diversity gain. However, the turbo coding is beneficial to decrease the bit error rate to enhanced data rate by coding gain. The simulation results prove that the coded transmission has better performance than un-coded transmission. It is also observed that a higher coding rate has better performance than low coding rates and un-coded transmissions.

The rest of the paper is organized as follows. Section II describes the system model. Section III explains about proposed OSTBC with turbo code scheme. Simulation results are given in Section IV and finally conclusion is summarized in Section V.

\section{SYSTEM MODEL}

The system model is designed to evaluate the OSTBC performance with and without turbo coding in MIMO channel. The transmitter design is based on bits coded by turbo code and inter-leaver combination to get coded gain, further it mapped into composite symbols through modulation method to transmit over channel. Lastly, these symbols are passed through OSTBC encoder to get antenna diversity.

\section{A. The Orthogonal Space Time Encoding}

The OSTBC encoding is based on Alamouti code for diversity transmission scheme [10]. The simplest diversity transmission metric is described as

$$
G=\left[\begin{array}{cc}
S_{1} & S_{2} \\
-S_{2}^{*} & S_{1}^{*}
\end{array}\right]
$$


where $\mathrm{S}$ are the data symbols that are transmitted by the $j$-th transmit antennas. And $(\cdot)^{*}$ denotes the complex conjugates which are transmitted from antennas.

Furthermore, the modulation techniques is used to transform bit to symbol with real or complex constellation $M$ $=2^{m}$ symbols, such as BPSK, QPSK and 8PSK for channel transmission.

\section{B. OSTBC MIMO Channel}

Assume that, the channel is flat fading for the $j$-th transmit antenna to the $i$-th receive antenna, where each transmitted symbol is multiplied by a randomly varying complex number. As the channel under consideration is usually a Rayleigh channel, the real and imaginary parts of $h_{i, j}$ are Gaussian distribution with mean $\mu_{h_{i, j}}=0$ and variance $\sigma_{h_{i, j}}^{2}=0.5$ [11]. At the receiver site, the noise $g$ has the Gaussian probability density function with

$$
P(n)=\frac{1}{\sqrt{2 \pi \sigma^{2}}} e \frac{-(n-\mu)^{2}}{2 \sigma^{2}}
$$

where $\mu=0$ and $\sigma^{2}=N_{o} / 2$.

The $h_{i, j}$ identifier at the receiver channel and the received signal on the antenna are defined as

$$
\left\{\begin{array}{l}
Z_{11}=h_{11} S_{1}+h_{12} S_{2}+g_{11}, \\
Z_{12}=h_{11} S_{2}^{*}+h_{12} S_{1}^{*}+g_{12}, \\
Z_{21}=h_{21} S_{1}+h_{22} S_{2}+g_{21}, \\
Z_{22}=h_{21} S_{2}^{*}+h_{22} S_{1}^{*}+g_{22}
\end{array}\right.
$$

where $h_{i j}$ is the among the $j$-th transmitter and $i$-th receiver antennas, $\mathrm{S}$ is represented as the data symbols, and the $g_{i j}$ is the additive noise between $i$-th receiver antenna at the $j$-th time slot.

When the $Z_{12}$ and $Z_{22}$ symbols are complex conjugates, then the representation (3) is shown in matrix form as

$$
\left[\begin{array}{l}
Z_{11} \\
Z_{21} \\
Z_{12}^{*} \\
Z_{22}^{*}
\end{array}\right]=\left[\begin{array}{ll}
h_{11} & h_{12} \\
h_{21} & h_{22} \\
h_{12}^{*} & -h_{11}^{*} \\
h_{22}^{*} & -h_{21}^{*}
\end{array}\right]\left[\begin{array}{l}
S_{1} \\
S_{2}
\end{array}\right]+\left[\begin{array}{l}
g_{11} \\
g_{21} \\
g_{12}^{*} \\
g_{22}^{*}
\end{array}\right]
$$

which is further expressed as

$$
Z=\mathrm{H}_{O} S+g
$$

where, the $g$ defends the channel noise and the $Z$ shows the output of MIMO channel, whereas, $\mathrm{H}_{O}$ represents the matrix of alike channel created through combination of OSTBC encoder and MIMO channel. Furthermore, the $\mathrm{H}_{O}$ is also expressed as an orthogonal matrix of entire channel recognition due to $\mathrm{H}_{O}^{H} \mathrm{H}_{O}=\|\mathrm{H}\|_{F}^{2} \mathrm{I}_{2}$, where $\|\cdot\|_{F}$ is the Frobenius norm and $H=\left[h_{i j}\right]$ is the channel matrix.

\section{The OSTBC Decoder}

An ideal channel state information (CSI) is assumed at the receiver, therefore the combining coefficients organism best selected uniformly with the complex conjugated channel matrix through utilization of maximal ratio combining (MRC) [12]-[14].

$$
\widetilde{\mathrm{S}}=\mathrm{H}_{O} \mathrm{Z}
$$

Furthermore, it can be expressed as

$$
\left[\begin{array}{c}
\tilde{\mathrm{S}}_{1} \\
\tilde{\mathrm{S}}_{2}
\end{array}\right]=\left[\begin{array}{cccc}
h_{11}^{*} & h_{21}^{*} & h_{12} & h_{22} \\
h_{12}^{*} & h_{22}^{*} & -h_{11} & -h_{21}
\end{array}\right]\left[\begin{array}{c}
Z_{11} \\
Z_{21} \\
Z_{12}^{*} \\
Z_{22}^{*}
\end{array}\right]
$$

Lastly, the collective symbols $\tilde{\mathrm{S}}_{1}$ and $\tilde{\mathrm{S}}_{2}$ are implemented with maximum likelihood (ML) decoder to retrieve the transmitted symbols. Even, if a pathway is sternly gray, then transmitted information can be recovered by other transmission paths with the help of equation (5) and (7) as

$$
\tilde{\mathrm{S}}=\mathrm{H}_{O}^{H} \mathrm{H}_{o} S+\mathrm{H}_{O}^{H} g=\|\mathrm{H}\|_{F}^{2} \mathrm{I}_{2} S+\mathrm{g}^{\prime}
$$

where $g^{\prime}$ have autocorrelation function and zero mean $E\left[g^{\prime} g^{\prime H}\right]=\sigma_{g}^{2}\|\mathrm{H}\|_{F}^{2} \mathrm{I}_{2}$. The symbols dissociated as:

$$
\left\{\begin{array}{l}
\tilde{\mathrm{S}}_{1}=\|Z\|_{F}^{2} S_{1}+\tilde{g}_{1}, \\
\tilde{\mathrm{S}}_{2}==\|\mathrm{H}\|_{F}^{2} S_{2}+\tilde{g}_{2}
\end{array}\right.
$$

where $Z$ is noisy and $R_{\mathrm{r}}$-dimensional received vector, $H$ is $R_{\mathrm{r}} \times$ $N_{\mathrm{A}}$ flat fading channel with entries $h_{i, j}$ that are assumed as i.i.d and $N_{\mathrm{A}}$ is number of transmit antennas and $R_{\mathrm{r}}$ represents the number of receiver antennas., Complex Gaussian random variable with zero-mean and unit variance $E\left[\left|h_{i, j}\right|^{2}\right]=1, S$ is $N_{\mathrm{A}}$-dimensional transmit vector $\in S^{N}$ which is assumed to be spatially uncorrelated and uniformly distributed, complex random vector process with zero-mean and variance $\sigma_{s}^{2}$ (i.e. $\left.R_{S S}=E\left[S S^{H}\right]=\sigma_{g}^{2} \mid N_{\mathrm{A}}\right), \mathrm{g}$ is the noise vector with dimension $R_{r} \times 1$ drawn from $\left(0, \sigma_{g}^{2}\right)$, or $\left.R g g=E\left[g g^{H}\right]=\sigma_{g}^{2} \mid R_{r}\right)$.

\section{OSTBC WITH TURBo CODE SCHEMES}

The proposed strategy is based on evaluation of OSTBC with and without turbo code over $4 \times 2$ and $4 \times 4$ MIMO channel. The proposed theme is based on turbo code with interleaving to get coding gain and OSTBC for diversity gain as shown in Fig. 1. The forward error correction (FEC) technique is used in proposed scheme to improve the data transmission and manage errors controls over erratic MIMO channel. One of the most frequently used FEC method is turbo code, which is renowned because of closely approaching 
Shannon channel capacity limit and coding gain with the help of inter-leaver. Turbo code designing with interleaving is discussed in [15], [16] with two recursive and systematic convolutional encoders (RSCC) and combined into parallel and split through pseudo random inter-leavers. The output of code matrix is defined as

$$
G(D)=\left[1 \frac{1+D+D^{3}}{1+D^{2}+D^{3}}\right]
$$

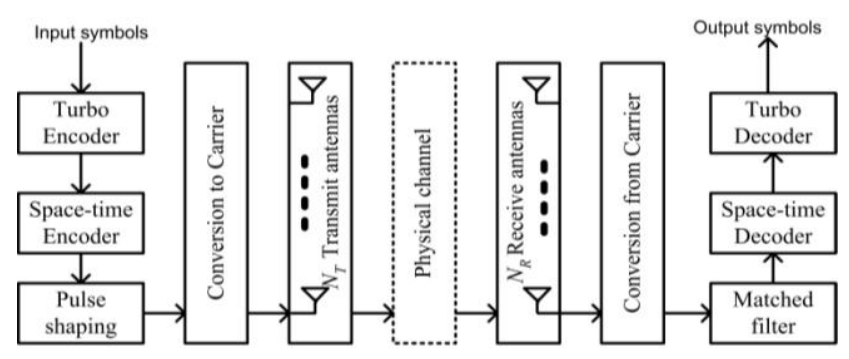

Fig. 1. A generic MIMO communication system.

where $1+D+D^{3}$ represents the feed-forward polynomial $\left(G_{f f}\right)$, the $1+D^{2}+D^{3}$ defines the feed-back polynomial $\left(G_{b f}\right)$ and the ratio of $\left(G_{f f}\right) /\left(G_{b f}\right)$ is useful to distinct the parity encoder.

The OSTBC technique is used to get diversity gain in MIMO transmission, which is fruitful to manage power ration and is also productive to retrieve appropriate information from strongest signal of $N$ received signals. As orthogonal STBC is a linear STBC symbols $S$, therefore it has the subsequent single chattels.

$$
\mathrm{S}^{H} \mathrm{~S}=\sum_{n=1}^{N}\left|\mathrm{~S}_{n}\right|^{2} \mathbf{I}
$$

where $i$-th row of S represents to the transmitted symbols $i$-th transmit antenna in $N$ transmission, whereas $j$-th column of $\mathrm{S}$ corresponds to the transmitted symbols at the same time by $N_{A}$ transmitted antennas at period $j$. The proposed system is designed according OSTBC with rate $1 / 2$ and $3 / 4$ for four transmit antennas in [17], while the code matrix is designed for equal power from all antennas in all time slots as:

$$
\begin{aligned}
S_{4,1 / 2} & =\left[\begin{array}{cccc}
S_{1} & S_{2} & S_{3} & S_{4} \\
-S_{2} & S_{1} & -S_{4} & S_{3} \\
-S_{3} & S_{4} & S_{1} & -S_{2} \\
-S_{4} & -S_{3} & S_{2} & S_{1} \\
S_{1}^{*} & S_{2}^{*} & S_{3}^{*} & S_{4}^{*} \\
-S_{2}^{*} & S_{1}^{*} & -S_{4}^{*} & S_{3}^{*} \\
-S_{3}^{*} & S_{4}^{*} & S_{1}^{*} & -S_{2}^{*} \\
-S_{4}^{*} & -S_{3}^{*} & S_{2}^{*} & S_{1}^{*}
\end{array}\right] \\
S_{4,3 / 4} & =\left[\begin{array}{cccc}
S_{1} & S_{2} & S_{3} & 0 \\
-S_{2}^{*} & S_{1}^{*} & 0 & S_{3} \\
-S_{3}^{*} & 0 & S_{1}^{*} & -S_{2} \\
0 & -S_{3}^{*} & S_{2}^{*} & S_{1}
\end{array}\right]
\end{aligned}
$$

The OSTBC data is further fed into the PSK constellation for $M=2^{m}$ symbols mapping, whereas the mapper read the $m$ $\times n_{t}$ coded bits and generate $n_{t}$ PSK symbols. Generally, it is formulated as: $k=\log _{2}(M)$.
The MIMO Channel uses array of multiple antenna at both sides, receiving and transmitting side. Consider a communication link that has $N_{A}$ number of antenna at Transmission and $R_{r}$ antenna at receivers. Let $N_{A}=R_{r}=n$. The transmit and receive signal vectors $s(t)$ and $r(t)$, respectively, can be defined as $s(t)=\left[s_{1}(t), s_{2}(t), \ldots . ., s_{n}(t)\right]^{T}, r(t)=\left[r_{1}(t), r_{2}(t), \ldots . ., r_{n}(t)\right]^{T}$.

The relationship between transmitters and receivers signal vector is given by:

$$
r(t)=H(t) S(t)+v(t)
$$

where $H(t)$ is known as the MIMO channel propagation matrix, and $v(t)$ is the AWGN additive noise. The matrix takes account of the multi-path propagation and can be expressed as:

$$
H=\left[\begin{array}{llll}
h_{11} & h_{12} & L & h_{1 j} \\
h_{21} & h_{22} & L & h_{2 j} \\
M & M & 0 & M \\
h_{11} & h_{i 2} & L & h_{i j}
\end{array}\right]_{R_{r} \times N_{A}}
$$

where $h_{i j}$ is the channel frequency response representing the complex path gain from transmit point $j$ to the receive point $i$, for $i=1,2, \ldots, R_{r}$ and $j=1,2, \ldots, N_{A}$.

The MIMO SNR is defined as

$$
S N R=\frac{n_{t} P_{s}}{\sigma_{n}^{2}}
$$

As SNR representation is symbols and bit based, which defined as

$$
S N R_{b i t}=\frac{S N R}{1 \mathrm{~d}|\mathrm{~A}|}
$$

where $|\mathrm{A}|$ consider the cardinality of the modulation arrangement.

The narrowband channel capacity for an $n \times n$ MIMO system is given by [18].

$$
C(\xi)=\log _{2} \operatorname{det}\left[\mathrm{I}_{n}+\frac{\xi}{n} \mathrm{HH}^{\mathrm{H}}\right]
$$

where $\xi$ represents the average signal-to-noise ratio at each receive point, $\operatorname{det}[x]$ represents the determinant operator, $I_{n}$ is the identity matrix of size $n \times n, \mathrm{H}$ is the normalised $n \times n$ propagation matrix such that indicates the hermitian operation defined as the conjugate transpose of $\mathrm{H}$.

$$
\sum_{j=1}^{n}\left(\left|h_{i j}\right|^{2}\right)=1
$$

At the receiving area, maximum likelihood (ML) decoding method is used because OSTBC insures the ML detection of transmitted symbols. The maximum likelihood detector (MLD) takes decision of the transmitted symbol $\left(S_{1}, S_{2}, \ldots S_{n}\right)$ rather than other decoding methods that are based on estimation of the channel coefficients $\left(h_{1}, h_{2}, \ldots, h_{n}\right)$ which are gathering through CSI. The estimates $S_{1}$ and $S_{2}$ are then sent 
to a MLD to calculate the transmitted symbols $S_{1}$ and $S_{2}$ correspondingly. The MLD conclusions for $1 / 2$ rates are expressed $S_{1}, S_{2}, S_{3}$ and $S_{4}$ by Eqs. (19), (20), (21) and (22) as:

$$
\begin{aligned}
& \mid\left[\sum _ { i = 1 } ^ { N _ { r } } \left(r_{i}^{(1)} h_{i, 1}^{*}+r_{i}^{*(2)} h_{i, 2}^{*}+r_{i}^{(3)} h_{i, 3}^{*}+r_{i}^{*(4)} h_{i, 4}^{*}\right.\right. \\
& \left.\left.+r_{i}^{(5)} h_{i, 1}^{*}+r_{i}^{*(6)} h_{i, 2}^{*}+r_{i}^{(7)} h_{i, 3}^{*}+r_{i}^{*(8)} h_{i, 4}^{*}\right)\right]-\left.S_{1}\right|^{2} \\
& +\xi\left|S_{1}\right|^{2} \\
& \mid\left[\mid \sum_{i=1}^{N_{r}}\left(r_{i}^{(1)} h_{i, 2}^{*}-r_{i}^{(2)} h_{i, 1}^{*}-r_{i}^{(3)} h_{i, 4}^{*}+r_{i}^{(4)} h_{i, 3}^{*}\right.\right. \\
& \left.\left.+r_{i}^{*(5)} h_{i, 2}-r_{i}^{*(6)} h_{i, 1}-r_{i}^{*(7)} h_{i, 4}+r_{i}^{*(8)} h_{i, 3}\right)\right]-\left.S_{2}\right|^{2} \\
& +\xi\left|S_{2}\right|^{2} \\
& \mid\left[\sum _ { i = 1 } ^ { N _ { r } } \left(r_{i}^{(1)} h_{i, 3}^{*}+r_{i}^{(2)} h_{i, 4}^{*}-r_{i}^{(3)} h_{i, 1}^{*}-r_{i}^{(4)} h_{i, 2}^{*}\right.\right. \\
& \left.\left.+r_{i}^{*(5)} h_{i, 3}+r_{i}^{*(6)} h_{i, 4}-r_{i}^{*(7)} h_{i, 1}-r_{i}^{*(8)} h_{i, 2}\right)\right]-\left.S_{3}\right|^{2} \\
& +\xi\left|S_{3}\right|^{2} \\
& +\xi\left|S_{4}\right|^{2} \\
& +\mid \sum_{i=1}^{N_{r}}\left(r_{i}^{(1)} h_{i, 4}^{*}-r_{i}^{(2)} h_{i, 3}^{*}-r_{i}^{(3)} h_{i, 2}^{*}+r_{i}^{(4)} h_{i, 1}^{*}\right. \\
& \left.\left.+r_{i}^{*(6)} h_{i, 3}-r_{i}^{*(7)} h_{i, 2}+r_{i}^{*(8)} h_{i, 1}\right)\right]-\left.S_{4}\right|^{2} \\
& +{ }^{(5)} \\
& +{ }^{*}{ }^{*} \\
&
\end{aligned}
$$

According to MLD decisions for $3 / 4$ rates, the following Eqs. (23), (24) and (25) are minimizing the overall values of $S_{1}, S_{2}$ and $S_{3}$ as:

$$
\begin{aligned}
& \mid\left[\sum_{i=1}^{N_{r}}\left(r_{i}^{(1)} h_{i, 1}^{*}+r_{i}^{*(2)} h_{i, 2}\right)+\frac{\left(r_{i}^{(4)}-r_{i}^{(3)}\right)\left(h_{i, 3}^{*}-h_{i, 4}^{*}\right)}{2}\right. \\
& \left.\quad-\frac{\left(r_{i}^{(3)}+r_{i}^{(4)}\right)^{*}\left(h_{i, 3}+h_{i, 4}\right)}{2}\right]-\left.S_{1}\right|^{2}+\xi\left|S_{1}\right|^{2} \\
& \mid\left[\sum_{i=1}^{N_{r}}\left(r_{i}^{(1)} h_{i, 2}^{*}-r_{i}^{*(2)} h_{i, 1}\right)+\frac{\left(r_{i}^{(4)}-r_{i}^{(3)}\right)\left(h_{i, 3}^{*}-h_{i, 4}^{*}\right)}{2}\right. \\
& \left.+\frac{\left(-r_{i}^{(3)}+r_{i}^{(4)}\right)^{*}\left(h_{i, 3}+h_{i, 4}\right)}{2}\right]-\left.S_{2}\right|^{2}+\xi\left|S_{2}\right|^{2} \\
& \mid\left[\mid \sum_{i=1}^{N_{r}}\left(\frac{\left(r_{i}^{(1)}-r_{i}^{(2)}\right) h_{i, 3}^{*}}{\sqrt{2}+\frac{\left(r_{i}^{(1)}-r_{i}^{(2)}\right) h_{i, 4}}{\sqrt{2}}}\right.\right. \\
& +\frac{r_{i}^{*(3)}\left(h_{i, 3}+h_{i, 4}\right)}{\sqrt{2}+\left.\frac{r_{i}^{*(4)}\left(h_{i, 1}+h_{i, 2}\right)}{\sqrt{2}}\right|_{3}}+\left.\xi\right|^{2}+\left.S_{3}\right|^{2}
\end{aligned}
$$

\section{Simulation AND Result Discussions}

Performance of OSTBC with turbo code $(1 / 2$ and $3 / 4$ coding rate) and without coding is evaluated in this simulation through various modulation schemes in $4 \times 2$ and $4 \times 4$ MIMO systems. The data signals are modulated by BPSK, QPSK and 8-PSK to transmit over Rayleigh fading channel with sampling frequency $(5 \mathrm{MHz})$ and doppler frequency $(10-\mathrm{Hz})$. The turbo recursive systematic convolutional code (RSC code) is used with coding rate $1 / 2$ and $3 / 4$ with frame length 10250. The number of transmit antennas are 4 and number of receive antennas are 2 and 4 . The graphs are plotted with Bit Error Rate (BER) versus Signal to Noise Ratio (SNR).

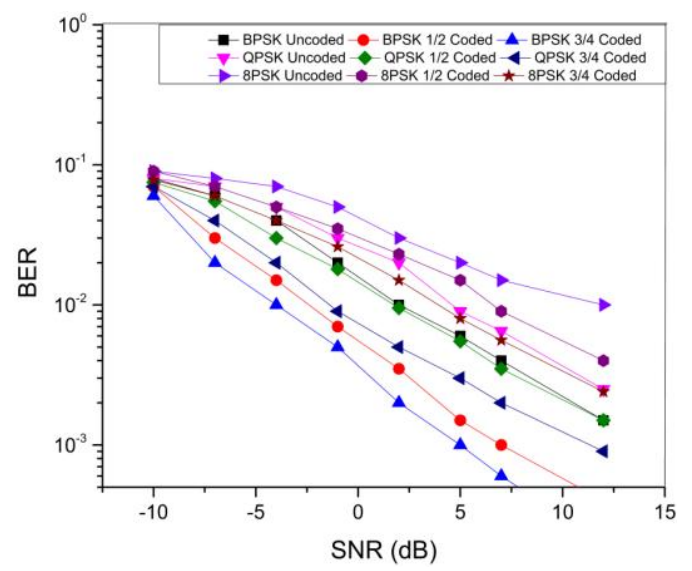

Fig. 2. OSTBC with and without coding performance over $4 \times 2$ MIMO.



Fig. 3. OSTBC with and without coding performance over $4 \times 4$ MIMO.

The result is revealed in Fig. 2 for $4 \times 2$ MIMO systems. As likely, for every diverse code blocks, performance get degrades as transmitter numbers of bit per symbols are increased. As shown in Fig. 2, the proposed method of $4 \times 2$ MIMO with $1 / 2$ and $3 / 4$ coded rate has better performance in $\mathrm{dB}$ as BPSK (4E-4 \& 2E-4 dB), QPSK (1.5E-03 \& 9E-4 dB) and 8-PSK (4E-03 \& 2.4E-3 dB) accordingly, than without turbo code scheme in which BPSK (1.5E-4 dB), QPSK (2.5E-3 dB) and 8PSK (1E-2 dB).

The outcome is shown in Fig. 3 for $4 \times 4$ MIMO systems. As expected, the performance order is same as in the case of $4 \times 2$ MIMO systems whereas the low bit per symbols has better performance than high bit per symbols due to less bit error rate. As shown in Fig. 3, the proposed $1 / 2$ and $3 / 4$ turbo code rates of $4 \times 4$ MIMO scheme has better performance for example, BPSK (1E-4 \& 9E-5 dB), QPSK (5E-4 \& 3E-4 dB) 
and 8PSK (1E-2 \& 5E-3 dB) accordingly, than without turbo code scheme in $\mathrm{dB}$ whereas BPSK $(4 \mathrm{E}-4 \mathrm{~dB})$, QPSK $(5 \mathrm{E}-3$ $\mathrm{dB})$ and $8 \mathrm{PSK}(2 \mathrm{E}-2 \mathrm{~dB})$. The deviation in $4 \times 2$ and $4 \times 4$ performance can be observed from Fig. 2 and Fig. 3 that the 4 $\times 4$ MIMO has better outcomes than $4 \times 2$ MIMO due to higher availability of diversity gain. The coding gain with diversity gain has the ability to overcome the bit error rate constraints.

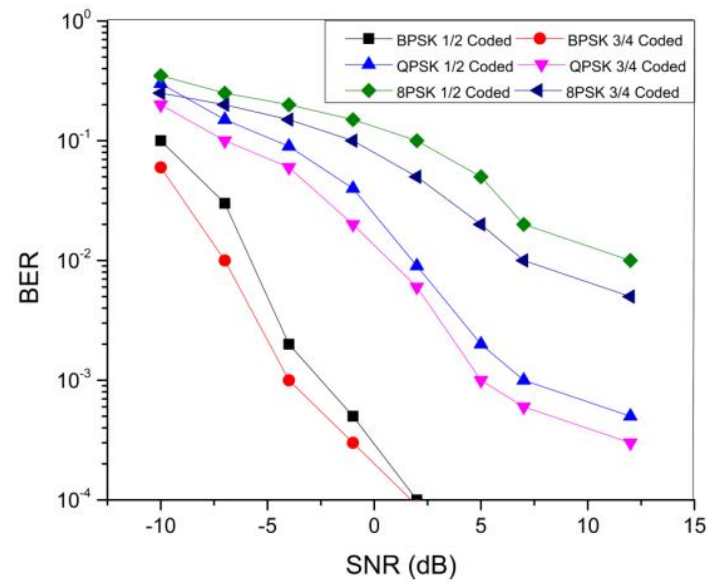

Fig. 4. OSTBC with Turbo coding $1 / 2$ and $3 / 4$ rates performance over $4 \times 2$ MIMO.

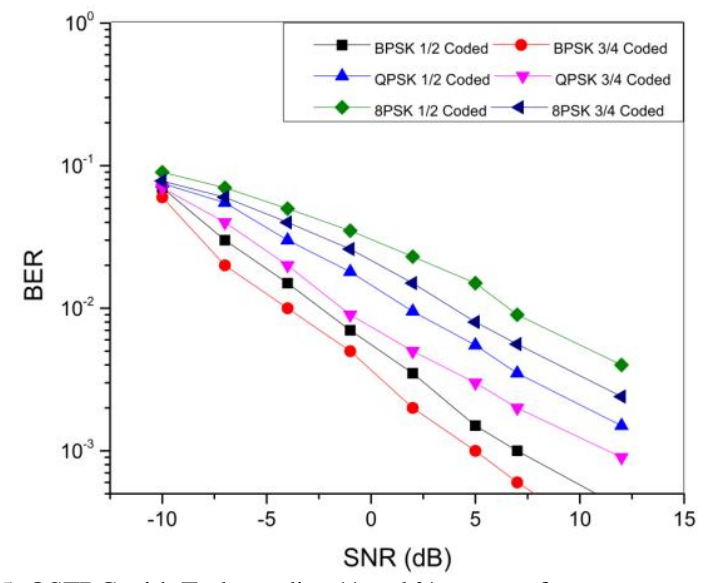

Fig. 5. OSTBC with Turbo coding $1 / 2$ and $3 / 4$ rates performance over $4 \times 4$ MIMO.

Furthermore, a comparison study between $1 / 2$ and $3 / 4$ turbo code rate is done to understand coding gain behavior with diversity gain $(\mathrm{OSTBC})$ in $4 \times 2$ and $4 \times 4$ MIMO networks. The comparison study is proved by simulation results that the higher coding gain rate $3 / 4$ has better performance than low coding gain rate $1 / 2$ in MIMO scenario. As show in Fig. 4 for 4 $\times 2$ MIMO that the $3 / 4$ coding rate has less bit error rate such as BPSK (2E-4 dB), QPSK (9E-4 dB) and 8PSK (2.4E-3 dB) than $1 / 2$ coding rate where BPSK (4E-4 dB), QPSK (1.5E-03 $\mathrm{dB})$ and 8 PSK $(4 \mathrm{E}-03 \mathrm{~dB})$. In the case of $4 \times 4$ MIMO the Fig. 5 represents the same behavior in which higher coding gain rate $3 / 4$ has better performance for example BPSK (9E-5 dB), QPSK $(3 \mathrm{E}-4 \mathrm{~dB})$ and 8 PSK $(5 \mathrm{E}-3 \mathrm{~dB})$ than low coding rate $1 / 2$ whereas BPSK (1E-4 dB), QPSK (5E-4 dB) and 8PSK (1E-2 $\mathrm{dB})$.

As overall conclusion, it is observed that the BPSK turbo code with $3 / 4$ rates is the best in terms of quality but on the tradeoff of the bandwidth. The quality degrades as modulation scheme increases from BPSK to QPSK to 8-PSK.
This is because when the number of modulated symbols increases from two to four to eight. The system complexity increases since the receiver now has to decide among greater number of symbols, the receiver while deciding makes error since there are increased number of symbols and this error causes the bit error rate to increase. The turbo code scheme is effective to improve the performance due to the ability of coding gain. The combination of coding gain by turbo coding and diversity gain via OSTBC has been fruitful to improve dramatically performance in MIMO systems. Another fact is also observed that the higher diversity gain $4 \times 4 \mathrm{MIMO}$ and higher coding gain rate $3 / 4$ has overall better performance than other coding and diversity gain schemes.

\section{CONCLUSIONS}

This paper evaluates the comprehensive comparative study of OSTB encoding with turbo code $(1 / 2$ and $3 / 4)$ and without turbo encoding for $4 \times 2$ and $4 \times 4$ MIMO systems. Diversity gain is useful to manage the power consumption and reduce the signal to noise ratio, which is explored through OSTBC. The coding gain is useful to reduce the bit error rate to improve data rate, which adopted by turbo encoding. At receiver sites, the optimal ML decoding is applied to extract the desired information. The results demonstrates that the proposed coded scheme has better performance than un-coded scheme, and also shows that the $3 / 4$ coding rates is better than both $1 / 2$ coding rate and without turbo encoding method

\section{REFERENCES}

[1] M. R Bhatnagar, R. Vishwanath, and V. Bhatnagar, "Performance analysis of space-time block codes in flat fading MIMO channels with offsets," EURASIP Journal on Wireless Communications and Networking, pp. 1-7, May 2007.

[2] H. Kaya et al., "Performance analysis of distributed turbo coded scheme with two ordered best relays," IET Communications, vol. 9, no. 5, pp. 638-648, 2015.

[3] S. L. Fu, X. G. Xia, and H. Q. Wang, "Recursive space-time trellis codes using differential encoding," IEEE Trans. on Information Theory, vol. 55, no. 2, pp. 531-546, Feb. 2009.

[4] B. Song, N. Kim, and P. Hyuncheol, "A binary space-time code for MIMO systems," IEEE Trans. on Wireless Communications, vol. 11, no. 4, pp. 1350-1357, April 2012.

[5] Y. X. Wang, J. Q. Wang, Z. B. Lu, and X. G. Liu, "Performance improvement of QO-STBC over time-selective channel for wireless network," Journal of Network and Computer Applications, vol. 36, no. 3, pp. 1018-1026, May 2013.

[6] X. B. Yu, W. T. Tan, S. H. Leung, Y. Rui, X. Yin, and X. S. Liu, "Discrete-rate adaptive modulation with optimum switching thresholds for space-ttime coded multiple-input multiple-output system with imperfect channel state information," IET Communications, vol. 7, no. 6, pp. 521-530, April 16, 2013.

[7] L. Hanzo et al., "Near-instantaneously adaptive HSDPA-style OFDM versus MC-CDMA transceivers for WIFI, WIMAX, and next-generation cellular systems," in Proc. the IEEE, Dec. 2007, vol. 95 , no. 12 , pp. 2368-2392.

[8] L. L. Yang, "Receiver multiuser diversity aided multi-stage minimum mean-square error detection for heavily loaded DS-CDMA and SDMA systems," IEEE Transactions on Communications, vol. 58, no. 12, pp. 3397-3404, December 2010.

[9] Y. Huang, W. Tang, H. Wei, J. Li, D. Wang, and X. Su, "On the performance of iterative receivers in massive MIMO systems with pilot contamination," in Proc. 2014 IEEE 9th Conference on Industrial Electronics and Applications, 2014, pp. 52-57.

[10] P. A. Lucian, "BER analysis of STBC codes for MIMO rayleigh flat fading channels," Telfor Journal, vol. 4, no. 2, pp. 78-82, 2012 
[11] N. Parveen and D. S. Venkateswarlu, "Multipath interferencee cancellation in MIMO mobile cellular System," International Journal of Distributed and Parallel Systems, vol. 3, no. 3, pp. 35-48, May 2012.

[12] F. J. Lopez-Martinez, J. T. Entrambasaguas et al., "Closed-form BER analysis of alamouti-MRC systems with ICSI in ricean fading channels," IEEE Communications Letters, vol. 15, no. 1, pp. 46-48, January 2011.

[13] Y. Z. Huang, F. S. Al-Qahtani, C. Zhong, Q. H. Wu, J. L. Wang, and H. M. Alnuweiri, "Cognitive MIMO relaying networks with primary user's interference and outdated channel state information," IEEE Transactions on Communications, vol. 62, no. 12, pp. 4241-4254, Dec. 2014

[14] S. Sugiura, L. Kong, S. Chen, et al., "Quasi-synchronous cooperative networks: A practical cooperative transmission protocol," IEEE Vehicular Technology Magazine, vol. 7, no. 4, pp. 66-76, Dec. 2012.

[15] N. Gresset, L. Brunel, and J. J. Boutros, "Space time coding techniques with bit-Interleaved coded modulations for MIMO block-fading channels," IEEE Trans. on Information Theory, vol. 54, no. 5, pp. 2156-2178, May 2008.

[16] P. Torres, C. Ludovic, and A. Gusmão, "Mobile communications with STBC and turbo codes," in Proc. 3rd Intl. Conf. on Innovations, Recent Trends and Challenges in Mechatronics, Mechanical Engineering and New High-Tech Products Development , 2011, vol. 3 , pp. 124-133.

[17] M. S. Hassan and K. Amis, "On the design of full-rate full-diversity space-time block codes for multiple-input-multiple-output systems with a turbo minimum mean square error equaliser at the receiver side," IET Communications, vol. 6, no. 18, pp. 3065-3074, Dec. 18 2012.

[18] S. R. Krishnamurthy and S. A. Jafar, "On the capacity of the finite field counterparts of wireless interference networks," IEEE Transactions on Information Theory, vol. 60, no. 7, pp. 4101-4124, July 2014.

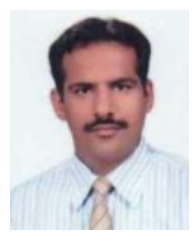

Naveed Ali Kaim Khani is a candidate of the PhD degree at School of Communication and Information Engineering, Dalian University of Technology, Dalian, China. He has a bachelor degree in electronics engineering from Sir Syed University of Engineering and Technology, Karachi, Pakistan and master's degree in computer and communication networks from Telecom \&
Management SudParis (ex INT), Evry, France. He has worked as a lecturer and the head of the Network Department from 2007 to 2009 at Universities of Engineering Science and Technology of Pakistan (UESTP-France), Karachi, Pakistan and also work as an assistant professor from 2009 to 2011 at COMSATS Institute of information Technology, Abbottabad, Pakistan His main research areas include, MIMO, beamforming, WiMax over GSM/GPRS network, physical layer of wireless network, energy efficient wireless network.

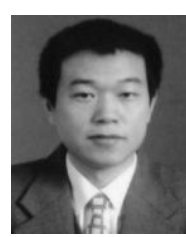

Zhe Chen received his B.S degree in electronics engineering, the M.S degree in signal and information processing, and the $\mathrm{PhD}$. degree in signal and information processing from Dalian university of Technology (DUT), Dalian, China, in 1996, 1999 and 2003 respectively. He joined the Department of electronics Engineering, DUT, as a lecturer in 2002, and become an associate professor in 2006. His research includes speech processing, image processing and wideband wireless communication.

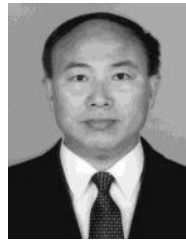

Fuliang Yin was born in Fushun city, Liaoning province, China, in 1962. He received the B.S. degree in electronic engineering and M.S. degree in communications and electronic systems from Dalian University of Technology (DUT), Dalian, China, in 1984 and 1987, respectively. He joined the Department of Electronic Engineering, DUT, as a lecturer in 1987 and became an associate professor in 1991. He has been a professor at DUT since 1994, and the dean of the School of Electronic and Information Engineering of DUT from 2000 to 2009. His research interests include digital signal processing, speech processing, image processing and broadband wireless communication. 